\title{
1’000'000 resolved points along a Brillouin distributed fibre sensor
}

\author{
Andrey Denisov*, Marcelo A. Soto, Luc Thévenaz \\ EPFL Swiss Federal Institute of Technology, Institute of Electrical Engineering, \\ Group for Fibre Optics SCI-STI-LT Station 11, CH-1015 Lausanne, Switzerland \\ *E-mail: andrey.denisov@epfl.ch
}

\begin{abstract}
The challenge to resolve a record of one million points along a single optical fibre is demonstrated using a Brillouin distributed fibre sensor. The proposed system is based on a phase-correlation technique combined with a temporal pump gating, making possible to detect a $14 \mathrm{~mm}$-long hot-spot located at the end of a $17.5 \mathrm{~km}$-long fibre. This corresponds to an increase by one order of magnitude in the number of points that a single system has resolved, representing a major breakthrough in the field. Specific issues arising when resolving such a large number of points are discussed.
\end{abstract}

Keywords: Fibre optics, optical fibre sensors, Brillouin scattering, distributed fibre sensor, nonlinear fibre optics.

\section{INTRODUCTION}

One of the parameters that uniquely characterise distributed sensing is the number of points that a single interrogation unit can resolve along an optical fibre, i.e. the quotient of the sensing range over the spatial resolution. Research efforts have been going essentially in two directions: $i$ ) extending the sensing range, and ii) improving the spatial resolution. The limitation to the spatial resolution imposed by the acoustic-wave response time has been overcome using dedicated configurations based on differential pulses ${ }^{1,2}$ or correlation-domain ${ }^{3-6}$, which have improved the spatial resolution down to $\mathrm{cm}$ or mm-scale. While Brillouin optical correlation-domain analysis (BOCDA) and reflectometry (BOCDR) offer a number of resolved points so far limited to a few thousands ${ }^{3}$, time-domain differential pulses and phase-modulated correlation-domain methods have demonstrated up to $300^{\prime} 000$ resolved points over a range of a few $\mathrm{km}$ of fibre ${ }^{5}(<5 \mathrm{~km})$.

On the other hand, the sensing range of classic Brillouin optical time-domain analysis (BOTDA) systems ${ }^{7}$ (typically limited to $30-50 \mathrm{~km}$ by the fibre attenuation, when using $1-5 \mathrm{~m}$ spatial resolution) has been substantially extended beyond $100 \mathrm{~km}^{8,9}$, using methods based on distributed Raman amplification, optical pulse coding, or a combination of them. This way, more than $100^{\prime} 000$ points have been demonstrated ${ }^{8,9}$, reaching even 240 '000 resolved points when both methods are combined with the differential pulse-width pair technique ${ }^{10}$.

Increasing the number of resolved points beyond a few hundred thousands, as demonstrated so far, turns out to be extremely challenging due to the weak Brillouin interaction and poor signal-to-noise ratio (SNR) resulting from very-high spatial resolution and/or very long sensing ranges ${ }^{7,11}$. Note that improving the spatial resolution in time-domain sensors reduces the Brillouin interaction and requires a broader detection bandwidth, both leading to a reduction in the SNR of the measurements. On the other hand, extending the sensing distance reduces significantly the sensor response as a consequence of the higher fibre attenuation that pump and probe signals experience during propagation ${ }^{7}$. But the challenge does not remain limited to a significant improvement of the SNR: any interfering signal must be kept at extremely low levels or eliminated by a very sharp and efficient filtering. Pump and probe powers have to remain below stringent limits to maintain interfering nonlinear effects at undetectable levels and to avoid severe pump depletion issues ${ }^{12}$.

In this paper a distributed optical fibre sensor capable of resolving more than 1'000'000 points is experimentally demonstrated for the first time to the best of our knowledge. This corresponds to one order of magnitude improvement in the number of resolved points over existing records for distributed fibre sensing, constituting a considerable leap forward in the field. The sensor is based on a phase-correlation technique combined with temporal pump gating, providing the possibility to reach $\mathrm{cm}$-scale spatial resolution with relatively low detection bandwidth (several $\mathrm{MHz}$ or lower), thus significantly reducing the noise level at the receiver. In particular, the high spatial resolution is verified by detecting a $14 \mathrm{~mm}$-long hot-spot at the end of a $17.5 \mathrm{~km}$-long standard single-mode fibre, using an electrical bandwidth of only $15 \mathrm{MHz}$. Fundamental practical limitations and specific issues arising from the large number of resolved points are described and discussed throughout the paper.

23rd International Conference on Optical Fibre Sensors, edited by José Miguel López-Higuera,

Julian Jones, Manuel López-Amo, José Luis Santos, Proc. of SPIE Vol. 9157, 9157D2

(C) 2014 SPIE · CCC code: 0277-786X/14/\$18 · doi: 10.1117/12.2071272 


\section{DESCRIPTION OF THE PROPOSED SYSTEM AND EXPERIMENTAL SETUP}

The sensor here described makes use of a phase-correlation technique ${ }^{4}$ in which pump and probe signals are phase-coded using a high-speed pseudo-random bit sequence (PRBS). This PRBS modulation allows Brillouin interaction to be efficient only at short correlation points (spanning over an interval given by the PRBS bit duration), while weak dynamic Brillouin gratings are generated randomly over other fibre locations. It has been recently demonstrated ${ }^{5}$ that the noise accumulated from the interaction with random gratings can be significantly reduced if the phase-coded pump is intensity gated with a square pulse, improving considerably the SNR of the system in comparison to the original phase-correlation scheme. The pulsed pump also makes it possible to measure simultaneously the Brillouin gain/loss spectrum for several correlation points along the fibre using standard time-domain detection. Since the pump pulse duration is independent of the spatial resolution and can be as long as hundreds of nanoseconds, a slow detection system can be used. However, a proper optimisation of the pump pulse is required: a short pulse actually needs a larger detection bandwidth, increasing the noise of the measurements, while a very long pulse increases the optical noise accumulated from weak random gratings generated along the pump pulse width. A preliminary analysis indicates that a pulse duration of 100-500 ns offers a good trade-off between the electrical noise at the receiver and the noise resulting from random gratings.

This paper targets the possibility to reach one million resolved points in a single system: this requires that the impact of the spatial resolution and sensing range on the SNR of the measurements has to be analysed to maximise the number of distinct points addressed along the fibre ${ }^{7,11}$. While the spatial resolution impacts linearly on the sensor response, increasing the number of resolved point by extending the sensing range turns out to be poorly efficient due to the huge SNR reduction imposed by the fibre exponential attenuation. The theoretical analysis presented in Ref. 11 indicates that an optimal number of resolved points can be found by a proper balance between the measuring distance and the spatial resolution, suggesting that one million points can be resolved by a single unit using an optimal fibre length lying in the 15-20 km range. Furthermore, the system design also needs to optimise the spectral width of the interacting waves in order to minimise the trace distortion that might result from optical filtering of the phase-modulated probe signal.

The proposed system is based on the experimental setup shown in Fig. 1. The laser diode output is guided through a phase modulator driven by a PRBS (applying a phase shift of 0 or $\pi$ ) with a bit duration of 145 ps (corresponding to full-width half-maximum correlation length of $\sim 14.5 \mathrm{~mm}$ ). The phase-modulated light is then split into two branches by an optical coupler. One branch is used to generate highpower pump pulses of $500 \mathrm{~ns}$ employing a semiconductor optical amplifier (SOA) and an erbium-doped fibre amplifier (EDFA). This phase-coded pump pulse is launched into a $17.5 \mathrm{~km}$-long sensing fibre through an optical circulator.

The other branch is used to generate twin probe waves with

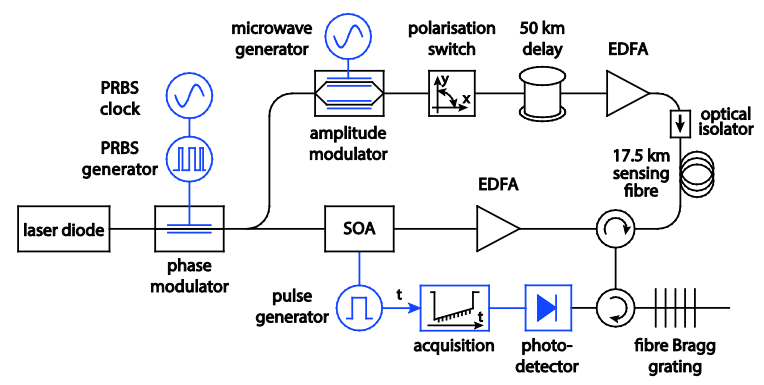

Figure 1. Experimental setup of phase-correlation Brillouin sensor, using time-domain low-bandwidth detection.

an intensity modulator driven by a microwave generator. Probe components are passed through a $50 \mathrm{~km}$-long delay fibre and a polarisation switch that is used to mitigate the polarisation dependence of the Brillouin interaction. The long delay is required to use high-order correlation peaks during the measurements (peaks from 33rd to 69th are allocated inside the sensing fibre), and can be implemented by any other method, for instance using proper synchronisation of the phase and amplitude modulations. An EDFA is then used to compensate for the losses in the delaying fibre. After interacting with the pump, the shorter-wavelength probe (experiencing Brillouin loss) is selected using a tuneable fibre Bragg grating (FBG) and directed onto a photo-detector followed by a $15 \mathrm{MHz}$ low-pass filter to reduce noise. Additional digital filtering is then used to further reduce noise and the signal bandwidth down to $2 \mathrm{MHz}$.

\section{RESULTS AND DISCUSSION}

Using time-domain acquisition and 2000 averaged traces, 37 correlation peaks are simultaneously measured for each pump-probe frequency offset. After acquiring such correlation peaks the clock frequency of the PRBS generator is changed to move correlation peaks to new positions. In order to scan the entire fibre the clock frequency is changed from $6.6 \mathrm{GHz}$ to $6.8 \mathrm{GHz}$; however, it is important to point out that although the spatial resolution depends on the clock frequency, such a frequency change shows a negligible effect, inducing less than $3 \%$ variation in the spatial resolution. Two time-domain traces (blue and red curves) having different clocks are shown in Fig. 2. The figure indicates that traces do not contain only the correlation peaks, but are also affected by other sources originated by as the non-ideal phase modu- 
lation and the random interaction outside of the correlation points. Although this background signal is strong, clear correlation peaks can be identified after subtracting the two measurements from each other, as depicted by the green curve in Fig. 2.

The Brillouin spectrum has been measured at randomly accessed points along a $17.5 \mathrm{~km}$ fibre. The extracted Brillouin frequency distribution as a function of the entire fibre length is shown in Fig. $3 \mathrm{a}$. Strong oscillations of the Brillouin frequency can be observed along the first $\mathrm{km}$ of fibre; however, it has been experimentally verified that these oscillations are actually real variations of the frequency, presumably resulting from additional strain in the fibre coiling. Fig. 3b shows that the standard deviation of the measured Brillouin frequency remains within $2 \mathrm{MHz}$ along the entire fibre and on average grows at a rate ruled by the fibre loss, indicating a frequency uncertainty of

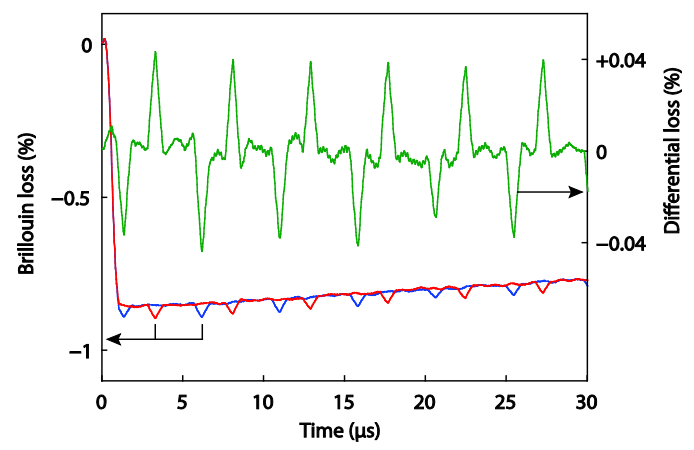

Figure 2. Time-domain traces for two PRBS clock frequencies (blue and red). Differential signal containing only responses of correlation peaks.
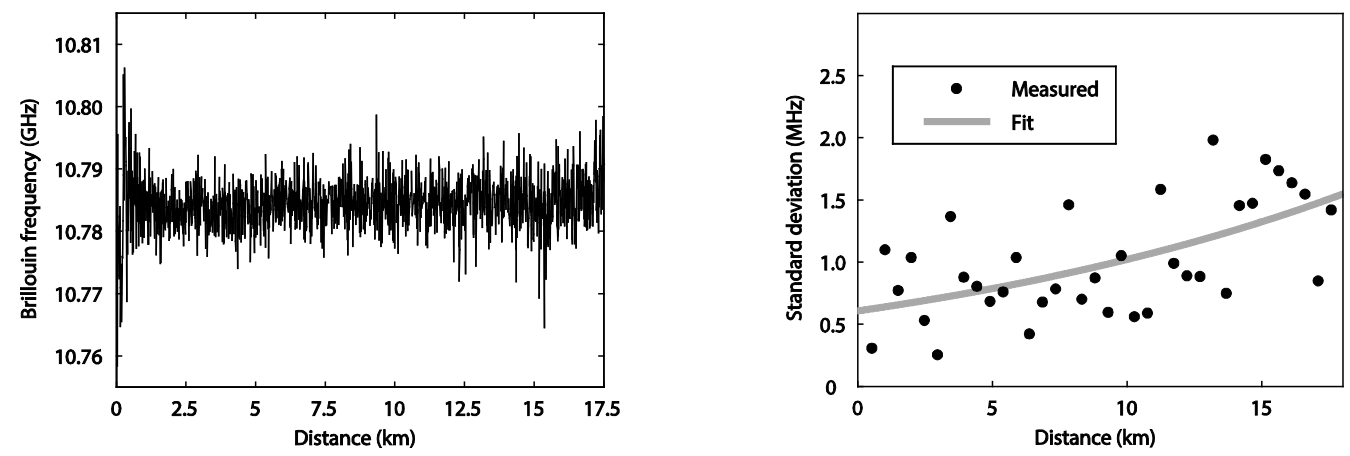

Figure 3. (a) Measured Brillouin frequency distribution along a $17.5 \mathrm{~km}$ fibre, and (b) respective standard deviation.

In order to verify experimentally the high spatial resolution of the system, a $14 \mathrm{~mm}$-long hotspot has been positioned and measured at the fibre distant end. The measured Brillouin gain spectrum and fitted Brillouin frequency are shown in Fig. 4. Results show that the hotspot can be clearly resolved, associated to an observable exponential heat transfer along the fibre. To the best of our knowledge this corresponds to the first demonstration of a distributed optical fibre sensor able to resolve more than one million points (exactly 1.25 million) along an optical fibre, constituting a considerable breakthrough in the field. The fact that the hotspot is resolved not as a single point, but as a short section of $\sim 7 \mathrm{~mm}$, also suggests that the actual spatial resolution is better than the expected $14 \mathrm{~mm}$. Further investigation is still required in order to evaluate the real spatial resolution and to discriminate it from the unavoidable heat transfer along the fibre.
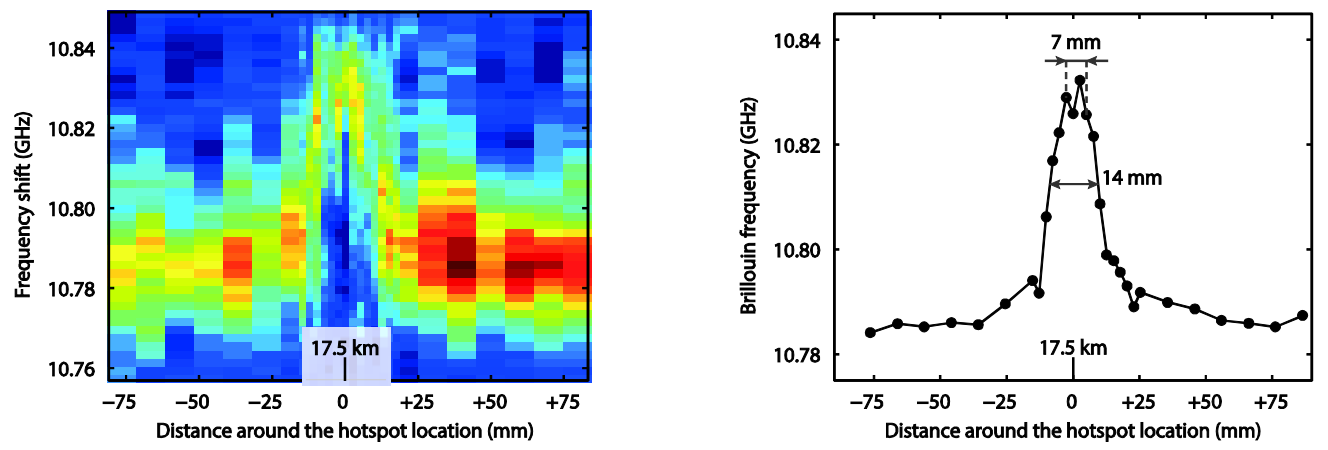

Figure 4. (a) Measured Brillouin gain and (b) Brillouin frequency around a $14 \mathrm{~mm}$ hotspot positioned at $17.5 \mathrm{~km}$ distance.

The increase of the number of points by one order of magnitude actually raises additional issues specific to distributed sensors showing such an extreme resolving power. First, a longer measurement time is inevitably expected to acquire such a large number of points, in particular with the use of correlation methods in which each fibre location has to be addressed separately. From this point of view, the time-domain acquisition of the proposed method offers the possibility 
to scan simultaneously the Brillouin gain at multiple fibre locations. Additionally, varying the PRBS bit duration, or turning off the phase modulation, the spatial resolution can be dynamically changed within centimetre or even metre scale. This way, an initial scan with long spatial resolution can be made with a further "zoom-in" at spotted regions of interest. With the use of specific electronics the correlation peaks can actually be positioned not only equidistantly, but rather at any required fibre position, resulting in a very versatile system.

Furthermore, considering that distributed optical fibre sensors make use of the speed of light in the fibre to convert timedomain into distance, the temperature dependence of the refractive index can no longer be neglected when one million points are resolved in a single optical fibre. The relative change of the refractive index with temperature is $10^{-5} \mathrm{~K}^{-1}$, and therefore a change of $0.1 \mathrm{~K}$ in the average temperature of the sensing fibre introduces a relative change in the time of flight of $10^{-6}$. It turns out that if the sensor is able to resolve $10^{6}$ points, this change in the time of flight introduces an error equal to the spatial resolution in positioning the measurements along the fibre length. It is therefore expected that non-negligible temperature variations along the fibre will certainly introduce significant uncertainty in the positioning precision of the sensor. Thus, a post-processing procedure should be considered to correct the local time of flight at each fibre location as a function of the measured point-by-point fibre temperature.

\section{CONCLUSION}

A spatial resolution of $14 \mathrm{~mm}$ over the distance of $17.5 \mathrm{~km}$ has been experimentally demonstrated in a distributed Brillouin fibre sensor, thus resolving more than 1'000'000 distinct points. This is the highest number of points ever demonstrated in a distributed fibre sensor, representing a major breakthrough in the field. The phase-correlation method is quite novel and there is still a margin for improving the performance of the system, potentially leading to faster measurements or a larger number of points. Yet, the problem of the uncertainty in positioning due to the change in ambient temperature will persist for any system with such a number of points and has to be addressed separately during the data processing.

We acknowledge the support from the Swiss National Science Foundation through the project 200021_134546 and the Swiss State Secretariat for Education, Research and Innovation (SERI) through the project COST C10.0093.

\section{REFERENCES}

[1] Foaleng, S. M., Tur, M., Beugnot, J.-C., Thevenaz, L., "High spatial and spectral resolution long-range sensing using Brillouin echoes," J. Lightwave Technol. 28 (20), 2993-3003 (2010).

[2] Dong, Y., Zhang, H., Chen, L. and Bao, X., " $2 \mathrm{~cm}$ spatial-resolution and $2 \mathrm{~km}$ range Brillouin optical fiber sensor using a transient differential pulse pair," Appl. Opt. 51 (9), 1229-1235 (2012).

[3] Mizuno, Y., He, Z. and Hotate, K., "Measurement range enlargement in Brillouin optical correlation-domain reflectometry based on double-modulation scheme," Opt. Express 18 (6), 5926-5933 (2010).

[4] Zadok, A., Antman, Y., Primerov, N., Denisov, A., Sancho, J. and Thevenaz, L., "Random-access distributed fiber sensing," Laser Photon. Rev.6, L1-L5 (2012).

[5] Denisov, A., Soto, M. A, and Thévenaz, L., "Time gated phase-correlation distributed Brillouin fibre sensor," Proc. SPIE 8794, Fifth European Workshop on Optical Fibre Sensors, 87943I (2013).

[6] Elooz, D., Antman, Y., Levanon, N, and Zadok, A., "High-resolution long-reach distributed Brillouin sensing based on combined time-domain and correlation-domain analysis," Opt. Express 22(6), 6453-6463 (2014).

[7] Soto, M. A. and Thévenaz, L., "Modeling and evaluating the performance of Brillouin distributed optical fiber sensors," Opt. Express 21(25), 31347-31366 (2013).

[8] Angulo-Vinuesa, X., Martin-Lopez, S., Corredera, P., Gonzalez-Herraez, M., "Raman-assisted Brillouin optical time-domain analysis with sub-meter resolution over 100 km," Opt. Express 20(11), 12147-12154 (2012).

[9] Soto, M. A., Taki, M., and Bolognini, G., "Simplex-Coded BOTDA Sensor Over 120-km SMF With 1-m Spatial Resolution Assisted by Optimized Bidirectional Raman Amplification,” IEEE Photon. Technol. Lett. 24(20), 1823-1826 (2012).

[10] Soto, M. A., Taki, M., Bolognini, G., and Di Pasquale, F., "Optimization of a DPP-BOTDA sensor with $25 \mathrm{~cm}$ spatial resolution over $60 \mathrm{~km}$ standard single-mode fiber using Simplex codes and optical pre-amplification," Opt. Express 20(7), 6860-6869 (2012).

[11] Soto, M. A. and Thévenaz, L., "Towards 1'000'000 resolved points in a distributed optical fibre sensor," Proc. SPIE 9157, 23rd International Conference on Optical Fiber Sensors OFS23, Invited paper (2014).

[12] Thévenaz, L., Foaleng Mafang, S. and Lin, J., "Effect of pulse depletion in a Brillouin optical time-domain analysis system," Opt. Express 21(12), 14017-14035 (2013). 